\title{
Détermination de la nature des hémoglobines chez 982 bovins africains et malgaches (taurins et zébus) par électrophorèse sur acétate de cellulose
}

\author{
par J. P. PETIT avec la Collaboration fechnique de P. BILLARD \\ Laboratoire de BIOCHIMIE I. E. M. V. T.
}

\begin{abstract}
RÉSUMÉ
La méthode décrite permet d'utiliser des échantillons de sang toial prélevés facilement ef se conservant bien, très loin du lieu où Ils sont recueillis, pour détermıner la nature des hémoglobines bovines. Après description du protocole d'électrophorèse sur acétate de cellulose, les résultats obtenus chez 982 bovins sont discutés avec leur interprétation statistique pour les 7 races bovines africaines et malgaches présentées. La fréquence des gènes $A$ et $B$ de l'hémoglobine a éré calculée pour chaque race, l'Indication de ses limites de confiance permettant une généralisation des résultałs.
\end{abstract}

\section{INTRODUCTION}

Le laboratoire ayant entrepris l'étude biochimique des raisons de la trypanotolérance par comparaison des caractéristiques sanguines de taurins (N'Dama) et de zébus (Gobra) a été amené à déterminer si des différences exıstaient dans la nature des hémoglobines. On a choisi l'électrophorèse de zone sur acétate de cellulose qui permet de façon simple d'excellentes séparations de protéines pourvu qu'on se tienne à des données rigoureusement définies. L'utilisation d'une cuve peu onéreuse facilite grandement la pratique des migrations et permet notamment une détermination élégante des groupes d'hémoglobine rencontrés chez un animal.

Le support de la migration, en acétate de cellulose, était relativement peu utilisé en raison de son prix. Son coût vient récemment de baisser de moitié permettant ainsi son emploi beaucoup plus large dans les laboratoires qu'ils soient de recherche ou d'analyse courante.
Le laboratorre de biochimie de l'I. E. M. V. T. propose une méthode simple dans sa mise en cuvre depuis la récolte de l'échantillon jusqu'à la détermination des hémoglobines caractéristiques d'un bovin, méthode applicable quel que soif l'éloignement entre le lieu où se trouve l'animal et le laboratoire d'analyse. Elle a été mise au point pour déterminer rapidement le nombre et la nature des hémoglobines contenues dans les hématies de certaines races de bovins africains. Cette première étape est doncessentiellement qualitative et elle a pour point de départ des échantillons de sang total recueillis sur papier filtre : l'acétate de cellulose utilisée selon les modalités décrites ici, permettant de déterminer la nature des hémoglobines malgré la présence des protéines plasmatiques.

\section{I. - PRÉLÈVEMENTS}

Les électrophorèses ont été effectuées en France tandis que les prélèvements étarent réalisés en 
brousse par les soins d'équipes en liaison avec le laboratoire et selon une technique analogue pour toutes les régions. Le sang était récolté à l'oreille sur papier filtre Whatman $3 \mathrm{MM}$ découpé préalablement au laboratoire, l'identification de l'animal se faisant directement sur ce même papier au moment du prélèvement. il est indispensable de ne pas rassembler les papiers filtre dans leur boîte de plastique avant qu'ils ne soient bien secs. La conservation des boîtes, hermétiquement closes, șans, réfrigérateur, laisse un délai d'un mois avant que les échantillons ne solent devenus inutilisables. En les mettant au réfrigérateur chaque fois qu'il est possible on peut considérablement allonger ce délai, et le porter à deux mois, pourvu que dès l'arrivée au laboratoire on conserve les boîtes, toujours closes, d̀ - $30^{\circ} \mathrm{C}$.

Des études sont poursuivies pour déterminer la durée exacte d'utilisation possible.

\section{II. - TECHNIQUE}

Elle a d'abord été mise au point pour des électrophorèses de méthémoglobine alcaline purifiée de telle sorte que les impuretés protéiques soient inférieures à 2 p. 1.000 . Puis on a comparé les résultałs obtenus avec des échantillons provenant de sang tatal recueilli sur papier filtre.

\section{a) Influence du lieu de dépôt.}

Les migrations de méthémoglobines purifiées sont excellentes quel que soit le lieu de dépôt des échantillons (fig. 1) et on peut considérer qu'on se trouve dans des conditions encore meilleures que lors de l'utilisation d'hémolysats frais car alors le taux de méthémoglobine est variable dans l'échantillon et pourrait en imposer pour un nouveau type d'hémoglobine. A cause de l'interférence des protéines plasmatiques, la différenciation des deux hémoglobines $A$ et $B$ est meilleure quand le dépôt est fait au $1 / 3$ de la bande vers la cathode lors de l'utilisation d'échantillons non purifiés, recueillis sur papier filtre (fig. 2).

\section{b) Solution tampon.} 0,09 .
Véronal acide.............. 2,76g

Véronal sodique ............ 15,40 g

Equ démınéralisée ............. 11 .

Pour obtenir exactement ce résultat il est important de n'effectuer les pesées qu'à poids constant. Cette solution est à utiliser fraîche, mais on peut la conserver 5 jours à $+40 \mathrm{C}$.

\section{c) Manipulations.}

\section{Préparation des bandes d'acéfate.}

1. Humidification.

Prendre chaque extrémité de la bande $(20 \times 150)$ avec des pinces en la maintenant légèrement courbe et poser la partie basse sur la solution tampon contenue dans une cuve peu profonde. Laisser retomber doucement les extrémités afin de ne pas emprisonner de bulles d'air. Après son humidification complète $(10 \mathrm{mn})$, l'immerger totalement dans la solution tampon. Retirer l'excès de tampon en plaçant la bande entre deux feuilles de buvard (aucun excès de tampon ne doit rester à la surface).

2. Dépôt des bandes dans lo cuve.

Dans chaque compartiment de la cuve (*) on met $500 \mathrm{ml}$ de tampon. On dépose à cheval sur une des baguettes de verre qui sert à la tension de l'acétate, un morceau de papier filtre Whatman no 1 qui est utilisé comme pont électrique en trempant largement dans le tampon et par son contact avec la bande d'acétate. On économise ainsi sur la longueur de la bonde d'acétate, qu'on tend en la serrant cntre deux baguettes de verre maintenues par un crochet attaché à l'extrémité d'un élastique fixé à la cuve. Dımensions du papier Whatman : $195 \times 35 \mathrm{~mm}$. On immerge le papier filtre sur $2,3 \mathrm{~mm}$ dans le tampon.

\section{$2^{\circ}$ Dépôt de l'échantillon.}

Al'aide d'une micropipette (**), d'un microlitre, on dépose cette quantité d'une solution d'hémoglobine à $2 \mathrm{~g} \mathrm{p}$. 100 au $1 / 3$ de la longueur de la bande du côté de la cathode. L'échantıllon doit être régulièrement réparti sur une largeur d'environ $8 \mathrm{~mm}$ pour une largeur de la bande

(*) Apelab à Bagneux.

(**) Pederson. 


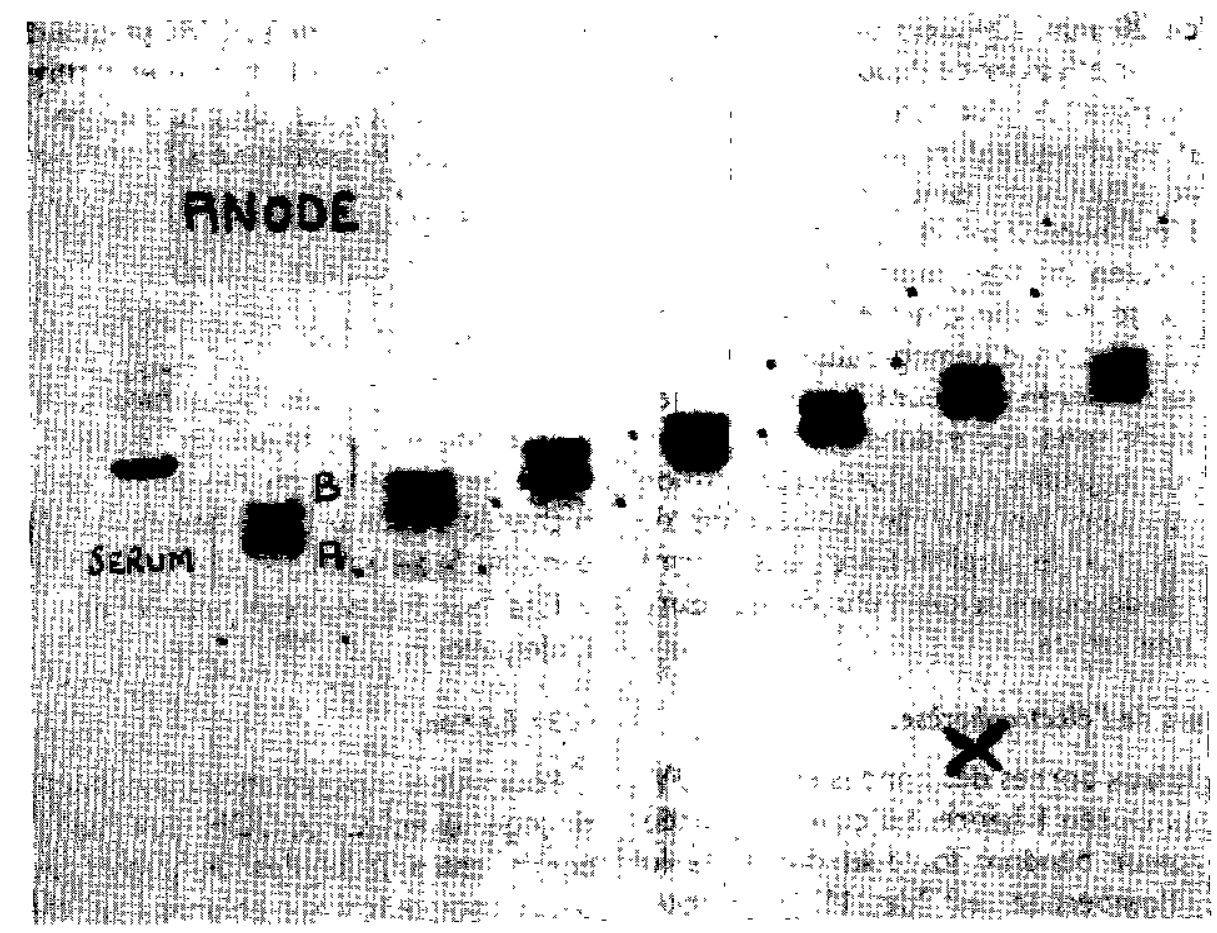

Fig. 1. - Influence de la position du dépôt de l'échantıllon de Méthémoglobıne purıfiée sur Ja migration. On voit nettement que la séparation des hémoglobınes $A$ et $B$ d'un même animal est la même quel que soit le lieu du dépôt dont on distıngue la trace sur chaque bande (au même nivecu que les deux points repère).

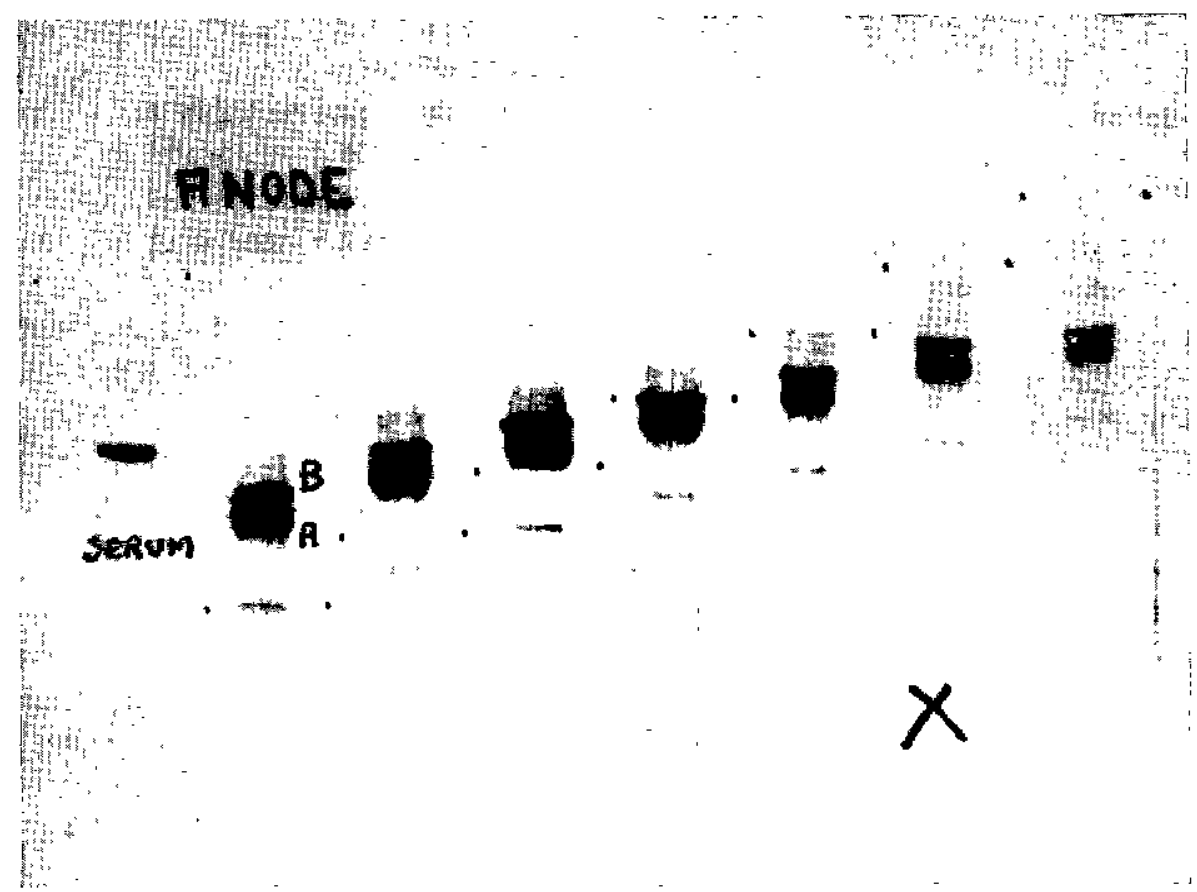

Fig. 2. - Influence de la position du dépôt de l'échantıllon provenant de sang total recueilli sur papier fiłtre. Pratiquement c'est quand le dépôt (Indiqué par deux points) est foit au $1 / 3$ vers la cathode que la séparation des hémogiobines $A$ et $B$ est la plus distincte. Dans cette positıon, on élimine au maximum les interférences dues aux protéines plasmatiques (bande $X$ ). 
d'acétate de $20 \mathrm{~mm}$. On doił prendre un soin particulier pour effectuer ce dépôt afin de ne pas altérer la surface de l'acétate et d'obtenir un mouvement extrêmement régulier de la main pour que le dépôt soit homogène sur toute sa largeur. II est nécessaire de souffler dans la pipette pour bien la vider mais il faut éviter la formation de toute bulle d'air à la surface de l'acétate. La solution d'hémoglobine à environ $2 \mathrm{~g} \mathrm{p} .100$ est préparée en éluant $3 \mathrm{~cm}^{2}$ de papier filtre imbibé de sang séché dans 0,20 à $0,25 \mathrm{ml}$ de tampon de migration. Le volume varie selon l'appréciation que l'on fait de la quantité de sang déposée sur le papier filtre. Les $3 \mathrm{~cm}^{2}$ sont finement découpés avant l'élution qui dure au moins une heure.

\section{Données de l'électrophorèse.}

Le voltage aux bornes des bandes est de $240 \mathrm{~V}$, soit $16 \mathrm{~V} / \mathrm{cm}$. Durée 1 heure. La cuve est placée au réfrigérateur pendant toute la durée de la migration, l'ampérage est de $11 \mathrm{~mA}$ pour 8 bandes $20 \times 150 \mathrm{~mm}$. Avec ces données, la condensation qui se produit sur le couvercle de la cuve n'est pas suffisante pour que des gouttes tombent sur l'acétate et perturbent les migrations.

\section{d) Interprétations.}

\section{Colorotion.}

Les bandes sont placées $10 \mathrm{mn}$ dans une solution colorante en évitant que l'échantilion touche un objet.

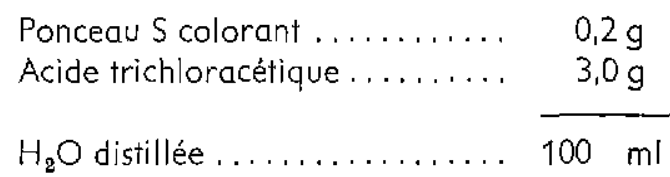

Les bandes sont décolorées avec $\mathrm{CH}_{3} \mathrm{COOH}$ glacial 5 p. 100 jusqu'à apparition de la surface blanche de l'acétate. Pendant toutes ces opérations les bandes sont dans un bac soumis à une agitation constante par balancement, un cycle dure 30 secondes.

\section{$2^{0}$ Diaphanisation.}

On éponge l'excès d'acide acétique puis on plonge les bandes dans une solution d'alcool à 95 p. 100. Agiter pendant $1 \mathrm{mn}$ puis bien essorer.
Placer chaque bande sur une plaque de verre un peu plus large que la bande et mettre la plaque dans la solution de diaphanisation 3 à $4 \mathrm{mn}$ (vérifier que la bande ne s'abîme pas).

p. 100 en volume

$\begin{array}{ll}\text { Cyclohexanone } \ldots \ldots \ldots \ldots \ldots & 33 \text { p. } 100 \\ \text { Ethanol à } 95 \text { p. } 100 \ldots \ldots \ldots \ldots & 67 \text { p. } 100\end{array}$

Faire partir l'excès de solution avec un agitateur entouré d'un tuyau en cooutchouc (ce qui empêche la formation des bulles d'air). Laisser sécher la baride à l'air libre ou à l'étuve à $100^{\circ}$ pendant 5 à $10 \mathrm{mn}$.

Ces bandes peuvent ensuite être passées à l'intégrateur.

\section{$3^{\circ}$ Elution.}

Pour une appréciation quantitative, après rinçage avec $\mathrm{CH}_{3} \mathrm{COOH}$, on falt sécher la bande entre 2 feuilles de papier buvard. On coupe chaque fraction colorée à mi-chemin entre les fractionnements. On coupe une surface analogue qui n'est pas colorée pour servir de témoin. On place chacun de ces morceaux dans des tubes différents contenant un volume identique de $\mathrm{NaOH} 0,1 \mathrm{~N}$; en général $2 \mathrm{ml}$ suffisent (ne pas dépasser $5 \mathrm{mi}$ ).

On agite le tube de façon intermittente jusqu'à extraction totale du colorant.

Ajouter une goutte de $\mathrm{CH}_{3} \mathrm{COOH}$ concentré par ml d'éluat de façon à ce que la coloration passe du pourpre au rose.

On passe les éluats dans des semi-microcuvettes et on mesure la densité optique à $540 \mathrm{~m} \mu$ (pour le rouge ponceau).

\section{III. — RÉSULTATS}

Les premiers essais ont été réalisés sur les animaux d'un élevage français de race Montbéliarde particulièrement pure, pour vérifier la valeur de la méthode. Ces hémoglobines ont été déterminées en même temps par électrophorèse en gel de polyacrylamide ef les résultats ont toujours été concordants avec ceux obtenus sur acétate de cellulose.

L'ensemble des déterminations figure dans le tableau I ; il représente le typage de l'hémoglobine chez 1.098 bovins. 
TABLEAU $\mathrm{N}^{\circ} \mathrm{I}$

Détermination de la nature des hëmoglobines chez guelques races bovines africaines et fréquence correspondante des génotypes

\begin{tabular}{|c|c|c|c|c|c|c|c|c|}
\hline \multirow{2}{*}{$R$ a c e s } & \multirow{2}{*}{ Localisation } & \multirow[t]{2}{*}{$\mathbb{N}$} & \multicolumn{3}{|c|}{$\begin{array}{c}\text { Nature des hémoglobines } \\
\text { déterminées }\end{array}$} & \multicolumn{3}{|c|}{$\begin{array}{l}\text { Fréquence des génotypes } \\
\text { d'hémoglobine correspondants }\end{array}$} \\
\hline & & & A & A et $B$ & B & AA & $\mathrm{AB}$ & BB \\
\hline Montbéliarde & Erance & 107 & 104 & 3 & 0 & 97,2 p. 100 & 2,8 p. 100 & $0 \quad p .100$ \\
\hline \multirow[t]{2}{*}{ N'dame } & $\begin{array}{c}\text { Bouaké } \\
\text { Côte d'Ivoire }\end{array}$ & 20 & 18 & 1 & 1 & 90 & " & 5 \\
\hline & Gebon & 51 & 51 & 0 & 0 & 100 & 0 & 0 \\
\hline Rénitelo & $\begin{array}{l}\text { Kianjasoa } \\
\text { Madagascar }\end{array}$ & 299 & 168 & 110 & 21 & $56,19 \quad 1$ & 36,79 & 7,02 \\
\hline $\begin{array}{l}\text { Zébu } \\
\text { Gobra }\end{array}$ & $\begin{array}{l}\text { Dara } \\
\text { Sénégal }\end{array}$ & 35 & 14 & 21 & 0 & 40 & 60 & 0 \\
\hline $\begin{array}{l}\text { Zébu } \\
\text { Brahman }\end{array}$ & $\begin{array}{l}\text { Kianjasoa } \\
\text { Madagascar }\end{array}$ & 42 & 8 & 21 & 13 & 19 & $"$ & 31 \\
\hline $\begin{array}{c}\text { Zêbu } \\
\text { malgache }\end{array}$ & Madagascar & 226 & 36 & 99 & 91 & $15,93 \quad "$ & 43,80 & $40,27 "$ \\
\hline $\begin{array}{l}\text { Zébu } \\
\text { soudan }\end{array}$ & $\begin{array}{l}\text { Bambari } \\
\text { R.C.A. }\end{array}$ & 67 & 27 & 32 & $B$ & $40,30 \quad 1$ & 47,76 & $11,94 "$ \\
\hline $\begin{array}{l}\text { Zébu } \\
\text { bororo }\end{array}$ & $\begin{array}{l}\text { Bambari } \\
\text { R.C.A. }\end{array}$ & 242 & 97 & 105 & 40 & 40,08 & 43,39 & $16,53 \cdots$ \\
\hline
\end{tabular}

\section{IV. - DISCUSSION}

La valeur technique de ces résultats est indiquée par les figures 3 et 4 qui montrent en particulier la régularité de la mıgration des hémaglobines $A$ ef $B$ d'une part et leur parfaite identité d'une race à l'autre et de taurin à zébu, ceci que l'hémoglobine soit pure ou mélangée au plasma.

Sur la figure 4 on peut voir la migration d'un mélange de méthémoglobine d'un taurin N'Dama $A B$ et d'un zébu Gobra $A B$, migration qui a le même aspect que celle d'échantillons provenant d'un seul animal de génotype $A B$ pour l'hémoglobine.

La valeur de ces résultats, quant à leur interprétation, dépend évidemment de l'importance de l'échantillonnage dans chaque race. Les intervalles de confiance à 5 p. 100, de la fréquence des génotypes d'hémoglobine sont réunis pour chaque race dans le tableau II.

La lecture des deux premiers tableaux indique qu'en première approximation on trouve plus d'hémoglobine $B$ chez les zébus que chez les taurins dans le cadre strict de cette enquête. D'un point de vue génétique, il est intéressant de calculer les fréquences correspondant aux gènes hémoglobine $A$ et $B$ pour pouvorr les comparer aux chiffres déjà connus et publiés (tableav III).

La rareté de l'hémoglobine $\mathrm{B}$ chez les N'Dama et son absence chez les Gobra méritent de retenir l'attention car chez toutes les autres races africaines et malgaches mentionnées ici ce n'est pas le cas. Les quelques rares N'Dama possédant une hémoglobine $B$ pourraient en hériter d'ancêtres possédant du sang d'importation (Jersey).

La pleine valıdité des intervalles de confiance indiqués repose sur le choix au hasard des animaux dont les échantillons nous parviennent, choix effectué parmi des populations très vastes, l'échantillon restant pełıt par rapport à ces populations. Cette dernière condition est parfautement vérifiée, seul le choix n'est pas entièrement au hasard, il est orienté par les possibilités de prélèvements rencontrés sur place. Mais ces contingences, si elles dọivent être 


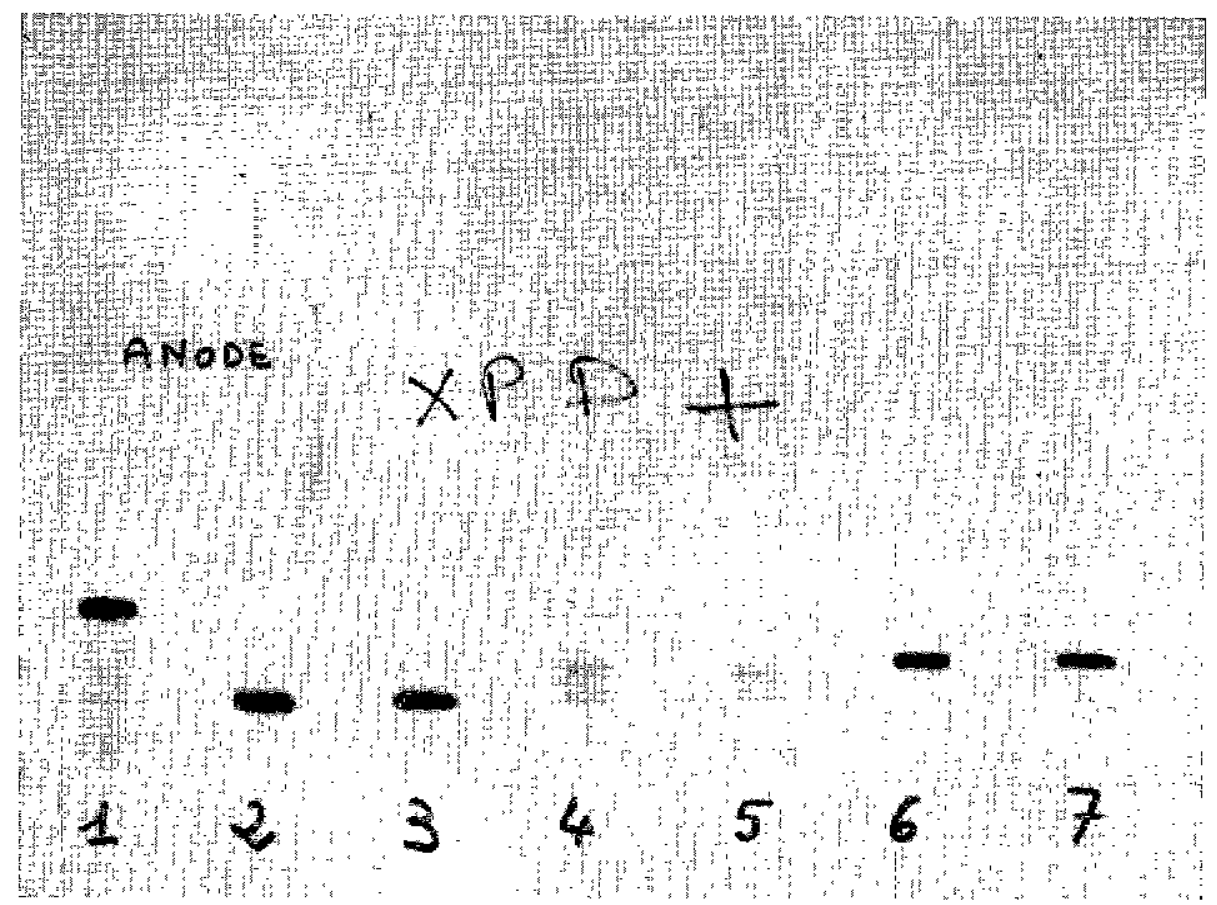

Fig. 3. - Régularité de la migration de diverses hémoglobines $A, A B$ et $B$. En 1 échantıllon de versatol normal qui figure à titre de témoin pour le repérage des mobılités. En 2 et 3 figurent des hémoglabines de type $A$, en 4 et 5 de type $A B$ et en 6 et 7 de type $B$, provenant d'animaux très différents.

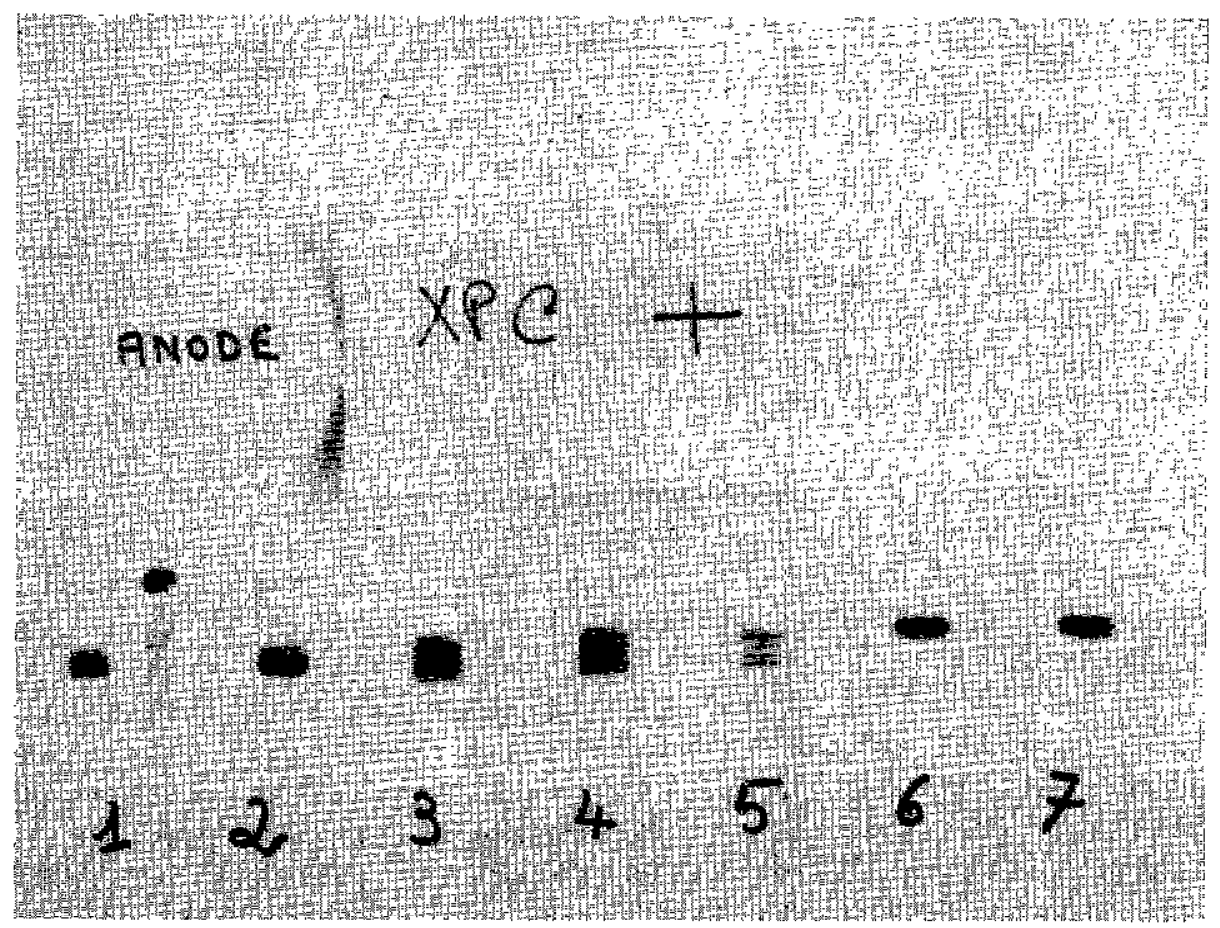

Fig. 4, - Jentité des hémoglobines d'origines diverses. En 4 l'échantillon déposé était constitué d'un mélange à parties égales de méthémoglobines pures appartenant à un gobra de génotype $A B$ ef à un $N$ 'dama de génotype $A B$ également.

En 1 en plus du versatol a été déposé un échantillon d'hémoglobine $A$ de gobra, en 2 hémoglobine $A$ de $N$ ' dama, en 3 hémoglobine $A B$ de gobra, en 5 hémoglobine $A B$ de $N$ 'dama, en 6 hémoglabine $B$ de gobra et en 7 hémoglobine de N'dama. 


\section{TABLEAU $N^{\circ}$ II}

Intervalles de confiance à $5 p .100$ des pourcentages de chaque gënotype d'hểmoglobine selon les races étudiẻes

\begin{tabular}{|c|c|c|c|c|c|c|c|c|c|c|c|}
\hline \multirow{2}{*}{$R$ a $c$ es } & \multicolumn{10}{|c|}{$\begin{array}{l}\text { Intervalle de confiance du pourcentage } \\
\text { des génotypes d'hêmoglobine }\end{array}$} & \multirow{2}{*}{$\mathrm{N}$} \\
\hline & \multicolumn{3}{|c|}{$\Lambda \mathrm{A}$} & \multicolumn{4}{|c|}{ A B } & \multicolumn{3}{|c|}{ B B } & \\
\hline Montbëliarde & \multicolumn{3}{|c|}{$89 \mathrm{p} \cdot 100-98 \mathrm{p} \cdot 100$} & \multicolumn{4}{|c|}{$2 \mathrm{p} \cdot 100-11 \mathrm{p} \cdot 100$} & & & & 107 \\
\hline $\mathrm{N}^{\prime}$ dama & $90 "$ & -9 & $"$ & 0 & it & $-2,7$ & & $0 \mathrm{p} \cdot 1$ & $0-2$ & $p .100$ & 71 \\
\hline Renitelo & $45 "$ & -5 & $"$ & 32 & $"$. & -43 & $\pi$ & 71 & -14 & $"$ & 299 \\
\hline Zëbu Gobra & $24 "$ & $-5 t$ & " & 42 & 11. & -76 & $"$ & & & & 35 \\
\hline Zébu Brahman & $9 "$ & -36 & " & 34 & $"$ & -66 & " & $17 "$ & -47 & $"$ & 42 \\
\hline Zébu malgache & $11 "$ & $-2 c$ & $"$ & 35 & $"$ & -52 & & 33 & -47 & $"$ & 226 \\
\hline Zëbu soudan & 28 & -52 & $"$ & 33 & $"$ & -57 & $"$ & 51 & $-2 C$ & $"$ & 67 \\
\hline Zébu bororo & $34 "$ & -47 & $"$ & 38 & $"$ " & -51 & $n$ & $10 "$ & -20 & $"$ & 242 \\
\hline
\end{tabular}

TABLEAU $N^{\circ}$ III

Fréquence des gènes correspondant à 1 'hêmoglobine $A$ et $B$ dans les échantillons des races étudiées avec leur intervalle de confiance à 5p.100

\begin{tabular}{|c|c|c|}
\hline \multirow{3}{*}{$R a c e s$} & \multicolumn{2}{|c|}{ Dans les échantillons êtudiés } \\
\hline & \multicolumn{2}{|c|}{ Fréquence du gène de l'hémoglobine } \\
\hline & A & $B$ \\
\hline Montbéliarde & $1 \pm 0,04$ & $0,014 \pm 0,05$ \\
\hline$N^{\prime} d a m a$ & $0,979 \pm 0,04$ & $0,024 \pm 0,05$ \\
\hline Rénitelo & $0,696 \pm 0,06$ & $0,240 \pm 0,05$ \\
\hline Zëbu Gobra & $0,700 \pm 0,160$ & $0,300 \pm 0,160$ \\
\hline Zêbu Brahman & $0,440 \pm 0,120$ & $0,560 \pm 0,150$ \\
\hline Zébu malgache & $0,378 \pm 0,045$ & $0,622 \pm 0,070$ \\
\hline Zëbu soudan & $0,642 \pm 0,120$ & $0,358 \pm 0,120$ \\
\hline Zëbu bororo & $0,617 \pm 0,07$ & $0,383 \pm 0,07$ \\
\hline
\end{tabular}

signalées n'orientent pas systématiquement le choix de tel ou tel animal, comme le ferait le fait de ne retenir que ceux qui se laissent facilement attraper, etc... ef donc ne biaisent pas les échantillons. Enfin, les petits pourcentages sont entachés d'une relativement trop grande erreur pour constituer des normes à retenır.

\section{V. - CONCLUSIONS}

Ces résultats ne sont qu'une contribution à la détermination biochimique des races. Groupés avec tous les autres caractères, ils doivent permettre aux zootechniciens d'étudier plus facilement les races pures et leurs croisements et également de rechercher des relations entre races différentes par des caractères biochimiques communs.

\section{REMERCIEMENTS}

Nous tenons à associer dans nos remerciements toutes les équipes qui onł participé à la récolte des échantillons : 
Mrs CLAIR et CROUAIL d̀ Bambari, CHAMBELLANT en R. C. A., COULOMB d̀ Bouake, DENIS au Sénégal, MONGODIN qui a assuré la liaison avec Mr GILIBERT du centre de Kianjasoa à Madagascar, CHOQUEL au Congo ef CHABEUF qui a assuré la liaison avec la plupart d'entre eux. De même nos remerciements vont à tous ceux qui ont permis l'expédition de ces échantıllons sur papier filtre et aux Directeurs des laboratoires Outre-Mer de I'I. E. M. V.T., Mrs ORUE et SERRES qui on facilité cette récolte. Que tous trouvent $\mathrm{ICl}$ l'expression de notre gratitude.

\section{SUMMARY}

Determination of the nature of the haemoglobins in 982 African and Malagasy cattle (taurines and zebus) by electrophoresis on cellulose acetate

Samples of tofal blood removed easily and with a good conservation state far from the place where they were collected, are used to determine the nature of cattle haemoglobins. After the description of protocol of electro-phoresis on cellulose acetate, the results obtained in 982 cattle are discussed in the light of their statistical interpretation, for the 7 African and Malagasy cattle breeds. The frequency of the gens $A$ and $B$ of haemoglobine has been evaluated for each breed ; a generalization of results has been made possible by the indication of their reliance limits.

\section{RESUMEN}

Determinación de la natura de las hemoglobinas en 982 bovinos africanos $y$ malgachos (faurinos y cebues) mediante electroforesis

sobre acelato de celulosa

El método descrito permite utilizar muestras de sangre total, facilmente tomadas e ya conservandose muy lejos del sitio donde se recogen, para determinar la natura de las hemoglobınas bovinas. Después de la descripción del proceso de electroforesis sobre acetato de celulosa, se discuten las resultados abtenidos en 982 bovinos con su interpretación estadística para las 7 razas bovinas y malgachas representadas. Se calcula la frecuencia de los genos A y $B$ de la hemoglobina para cada raza, permitiendo una generalización de los resultados sus límites de aseguramiento.

\section{BIBLIOGRAPHIE}

BALAKRISHNAN (C. R.) ef NAIR (P. G.). Haemoglobin polymorphism in Indian catile. Indian J. Genet. Pl. Breed, 1966, 26 A (Spec. Symposium No) : 374-85.

BRAEND (M.), EFREMOV (G.) et RAASTAD (A.). - Genetics of bovine hemoglobin D. Hereditos, 1966, $54: 255-59$.

DEMARET (M.). — Etude d'une ultramicrométhode d'électrophorèse sur acétate de cellulose (Microzone électrophorèse). Ann. Bıol. clin., 1966, 24 (3-4) : 369-82.

DEMARET (M.) et HEUDSEN (A.). - Dosage de l'hémoglobine $A_{2}$ après séparation électrophorétique en gel d'amidon et sur acétate de cellulose en « mlcrozone électrophorèse », Ann. Biol. clin., 1966, 24 (3-4) : 383-92.

MULLER (M.), FONTAINE (G.), MULLER (P.). - Intérêt médico-légal de l'étude électrophorétique de l'hémoglobine. Arch. Inst. Méd. lég. Méd. Soc. Lille, 1966, 203-229 (59 réf.).

NAIK (S. N.) et SANGHV! (L. D.), - Haemoglobin Khillari, a new variant in Indian cattle. Indian vet. J., 1966, 43 : 789. 92. 
PINFIELD (A. S.) et RODGERSON (D. O.). Quantitation of $A_{2}$ hemoglobin by electrophoresis on cellulosa acetate. Clin. Chem. U.S. A., 1966, 12 (12) : 883-6.

SEN (A.), ROY (D.), BHATTACHARYA (S.) et DEB (N. C.). - Haemoglobins of Indian Zebu cattle and the India buffalo. J. Anim. Sci., 1966, $25:$ 445-48.

AICARDI (G.). - Possibilita di impiego dell' acetato di cellulosa per la determinazione della frazione emoglobinica $A_{2}$. Boll. Soc. itol. Biol. sper., 1965, 41 (19) : 115153.

BRIERE (R. O.), GOLIAS (T.) et BATSAKIS (J. G.). - Rapid qualitative and quantitative haemoglobin fractionation. Cellulose acetate electrophoresis. Amer. J. clin. Pathol., 1965, 44 (6) : 695-701.

MARENGO-ROWE (A. 1.). - Rapid electrophoresis and quantitation of haemoglobins on cellulose acetate. J. clin. Pathol. G. B., 1965, $18(6): 790-2$.

MIKLE (S.). - (Polymorphlsme de I'hémoglobine chez les bovins). Revue Roum. Biol. Ser, Zool, 1965, 10 : 273-79 (En russe).

NAIK (S. N.) et SANGHVI (L. D.). - A new haemoglobin variant in zebu cattle. In blood groups of animols. Proc. 9th Eur. Anim. Blood grp. conf. Prague, 1964: 295-99 (publié en 1965).

NAIK (S. N.), SUKUMARAN (P. K.) et SANGHVI (L. D.). - A note on blood groups and haemoglobin variants in zebu cattle. Anim. proc., 1965, $7: 275-77$.

CARR (W. R.). - The hemoglobins of indigenous breeds of cartle in Central Africa. Rhod. J. agric. Res., 1964, 2 : 93-94.

BARTLETT (R. C.). - Rapid cellulose acetate electrophoresis II. Quantitative hemoglobin fractionation. Clin. chem. U. S. A., 1963, 9 (3) : 325-29. 